\title{
Caractéristiques des protéines texturées de lait
}

\author{
par \\ G. Ozimek, S. Poznanski, Z. Smietana \\ et J. SZPENDOWSKI*
}

\section{INTRODUCTION}

Au cours des dernières années, on a observé un intérêt de plus en plus vif pour des protéines nouvelles, dites non-conventionnelles, en vue de leur utilisation dans l'alimentation de l'homme. Un des procédés pour compenser, au moins partiellement, la déficience actuelle en protéines animales, ainsi que pour satisfaire la demande toujours grandissante en viande et en produits carnés, consiste à utiliser diverses préparations protéiques comme additifs et succédanés.

En considération de tout cela, et tenant compte des réserves considérables en protéines de lait dans de nombreux pays du monde, une technique de production des protéines texturées a été mise au point à l'Institut du Génie et de la Biotechnologie alimentaire de l'Université Agrotechnique d'Olsztyn, en utilisant le procédé de fibrisation thermoplastique [14]. Produites par ce procédé, les protéines texturées de lait possèdent une structure fibreuse, résistante à l'action de la température de stérilisation.

\section{PROTOCOLE EXPERIMENTAL}

On a effectué l'estimation de la valeur nutritive de la protéine de la préparation texturée.

Pour cela, on a procédé aux déterminations suivantes :

— teneur en protéine totale, par le procédé de Kjeldahl [1] ;

\footnotetext{
* Institut du Génie et de la Biotechnologie Alimentaire, Université Agrotechnique d'Olsztyn (Pologne).
} 
- composition en acides aminés, par la méthode de Moore et al. $[11,17]$ à l'aide d'un analyseur automatique J.L.C.-6 AH de la Compagnie Joel, après hydrolyse des protéines dans $6 \mathrm{n} / \mathrm{HCl}$.

La teneur en acides aminés sulfuriques (méthionine, cystéine et cystine) était déterminée selon la méthode rapportée par Schram et al. [15]. La teneur en tryptophane était déterminée par la méthode colorimétrique décrite par Opienska-Blauth et al. [12] après hydrolyse des protéines dans $5 \mathrm{n} / \mathrm{NaOH}$.

A partir de la composition en acides aminés, l'indice d'un acide aminé limitant, d'après Mitchell et Block [10], ainsi que l'indice intégré des acides aminés exogènes, d'après Oser [13], étaient déterminés. Le blanc d'œuf de poule, entier, ayant la composition en acides aminés, décrite par FAO (Report of Joint FAO/WHO expert Group : Protein Requirement, 1965, $n^{\circ} 37$ ), servait d'étalon. Le coefficient d'utilisation de la protéine nette, NPU, était aussi déterminé en utilisant la méthode de Miller et Bender [9], ainsi que le coefficient de l'efficacité de croissance de la protéine, PER, d'après Campbell [2] et le coefficient de digestion apparente, selon la méthode de bilan.

Outre cela, des protéines texturées de lait étaient examinées au microscope, et les propriétés physico-chimiques du texturat étaient définies, en prenant en considération l'influence de quelques facteurs du milieu.

La caractéristique physico-chimique comprenait les déterminations suivantes :

- teneur en protéines solubles, par la méthode d'Inklaar et Fortuin [6], à $\mathrm{pH}$ du milieu, différencié (de 1,0 à 10,0 ) et à concentration en $\mathrm{NaCl}$, variée ;

- absorption d'eau, par la méthode de Janicki et Walczak [7], à concentrations variées de $\mathrm{NaCl}$;

- aptitude à émulsionner la matière grasse, par la méthode de Swift et al. [19], modifiée par Kwasniewska et al. [8].

L'aptitude des protéines à émulsionner était déterminée à une teneur variée de la solution en protéines (de 9 à $350 \mathrm{mg}$ ) $10 \mathrm{ml}$ à $\mathrm{pH}$ du milieu allant (de 2 à 9,0) et à la concentration en $\mathrm{NaCl}$ (de 0,0 à $1 \mathrm{M}$ ).

\section{RESULTATS ET DISCUSSION concernant la valeur nutritive des protéines texturées}

L'analyse de la composition en acides aminés (tab. 1) a mis en évidence, que cette protéine se caractérisait par une teneur élevée en lysine $-8,16 \mathrm{~g} / 16 \mathrm{~g} \mathrm{~N}$. La teneur en tryptophane était de 1,15 g/ $16 \mathrm{~g} \mathrm{~N}$ et celle en acides aminés sulfuriques $-3,69 \mathrm{~g} / 16 \mathrm{~g} \mathrm{~N}$. Les acides 


\section{TABLEAU 1}

Composition en acides aminés et indices de la valeur nutritive de la protéine du texturat*

\begin{tabular}{l|c}
\multicolumn{1}{c|}{ Acides aminés $(\mathrm{g} / 16 \mathrm{~g} \mathrm{~N})$} & Texturat \\
\hline & \\
Acides aminés indispensables : & \\
Isoleucine & 4,22 \\
Leucine & 9,03 \\
Lysine & 8,16 \\
Méthionine & 2,93 \\
Cystéine et cystine & 0,76 \\
Acides aminés sulfuriques en total & 3,69 \\
Phénilalanine & 4,68 \\
Tyrosine & 5,42 \\
Acides aminés aromatiques en total & 10,10 \\
Thréonine & 3,76 \\
Tryptophane & 1,15 \\
Valine & 5,64 \\
& \\
\hline Indices : & \\
CS & 64,7 \\
EAA & \\
& \\
& \\
\hline
\end{tabular}

* Teneur en protéine de la préparation étudiée $=29,97$ p. 100.

aminés limitants sont des acides aminés sulfuriques - CS est au niveau de 64,7. L'indice d'Oser est aussi élevé, il atteint 83,1.

Des expérimentations faites sur les animaux ont montré que la valeur biologique de la protéine de la préparation, exprimée par des indices PER et NPU, est élevée, et elle est comparable aux valeurs de ces indices pour la caséine (tab. 2).

Le coefficient de PER est de 2,57 pour la protéine du texturat et de 2,59 pour la caséine, celui de N.PU - 61,2 p. 100 au regard de 61,6 pour la caséine. Le coefficient de digestibilité apparente de la protéine du texturat est au niveau de 87,14.

Des résultats, obtenus par des méthodes chimiques et biologiques, montrent la qualité de la protéine de texturat. Les protéines texturées de lait se caractérisent par une teneur plus élevée en acides aminés sulfuriques, par comparaison avec les protéines texturées de soja, ainsi que par une teneur plus élevée en lysine. Hamdy [3] rapporte 
TABLEAU 2

Valeur nutritive des protéines texturées du lait et de la caséine

\begin{tabular}{|c|c|c|c|c|c|c|c|c|}
\hline \multirow{2}{*}{\multicolumn{2}{|c|}{$\begin{array}{l}\text { Type de } \\
\text { protéine }\end{array}$}} & \multirow{2}{*}{$\begin{array}{l}\text { Augmentation du poids } \\
\text { de corps de rats } \\
(\mathrm{g} / 28 \mathrm{j})\end{array}$} & \multirow{2}{*}{$\begin{array}{l}\text { Consommation } \\
\text { de la protéine } \\
(\mathrm{g} / 28 \mathrm{j})\end{array}$} & \multirow{2}{*}{ PER } & \multirow{2}{*}{$\begin{array}{l}\text { NPU } \\
\text { (p. 100) }\end{array}$} & \multicolumn{2}{|c|}{ Protéine (g) } & \multirow{2}{*}{$\begin{array}{l}\text { Coefficient de } \\
\text { digestibilité } \\
\text { (p. 100) }\end{array}$} \\
\hline & & & & & & consom- & absorbée & \\
\hline \multirow[t]{2}{*}{$\begin{array}{l}1 \\
2 \\
3 \\
4 \\
5 \\
6 \\
7 \\
8\end{array}$} & 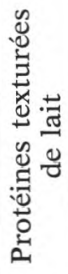 & $\begin{array}{l}83,0 \\
77,8 \\
79,6 \\
76,0 \\
80,3 \\
75,3 \\
81,6 \\
80,9\end{array}$ & $\begin{array}{l}31,56 \\
30,16 \\
30,66 \\
30,12 \\
31,08 \\
29,87 \\
31,45 \\
31,60\end{array}$ & $\begin{array}{l}2,63 \\
2,58 \\
2,60 \\
2,52 \\
2,58 \\
2,52 \\
2,59 \\
2,56\end{array}$ & $\begin{array}{l}61,6 \\
60,8\end{array}$ & $\begin{array}{r}8,53 \\
9,42 \\
8,40 \\
10,23 \\
10,45 \\
9,79 \\
10,37 \\
11,10\end{array}$ & $\begin{array}{l}7,29 \\
8,26 \\
7,31 \\
8,94 \\
9,03 \\
8,71 \\
9,01 \\
9,75\end{array}$ & $\begin{array}{l}85,5 \\
87,8 \\
87,1 \\
87,5 \\
86,4 \\
88,1 \\
86,9 \\
87,9\end{array}$ \\
\hline & $\overline{\mathrm{x}}$ & $79,31 \pm 1,67$ & $30,81 \pm 0,82$ & $2,57 \pm 0,111$ & 61,2 & $9,79 \pm 0,30$ & $8,54 \pm 0,87$ & $87,14 \pm 0,71$ \\
\hline \multirow[t]{2}{*}{$\begin{array}{l}1 \\
2 \\
3 \\
4 \\
5 \\
6 \\
7 \\
8\end{array}$} & 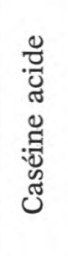 & $\begin{array}{l}76,4 \\
74,9 \\
78,6 \\
79,4 \\
80,2 \\
78,8 \\
79,6 \\
79,0\end{array}$ & $\begin{array}{l}29,61 \\
29,49 \\
30,23 \\
30,31 \\
30,49 \\
30,42 \\
30,62 \\
30,74\end{array}$ & $\begin{array}{l}2,58 \\
2,54 \\
2,60 \\
2,62 \\
2,63 \\
2,59 \\
2,60 \\
2,57\end{array}$ & $\begin{array}{l}61,9 \\
61,3\end{array}$ & & & \\
\hline & $\overline{\mathrm{x}}$ & $78,4 \pm 1,79$ & $30,24 \pm 0,46$ & $2,59 \pm 0,028$ & 61,6 & & & \\
\hline
\end{tabular}



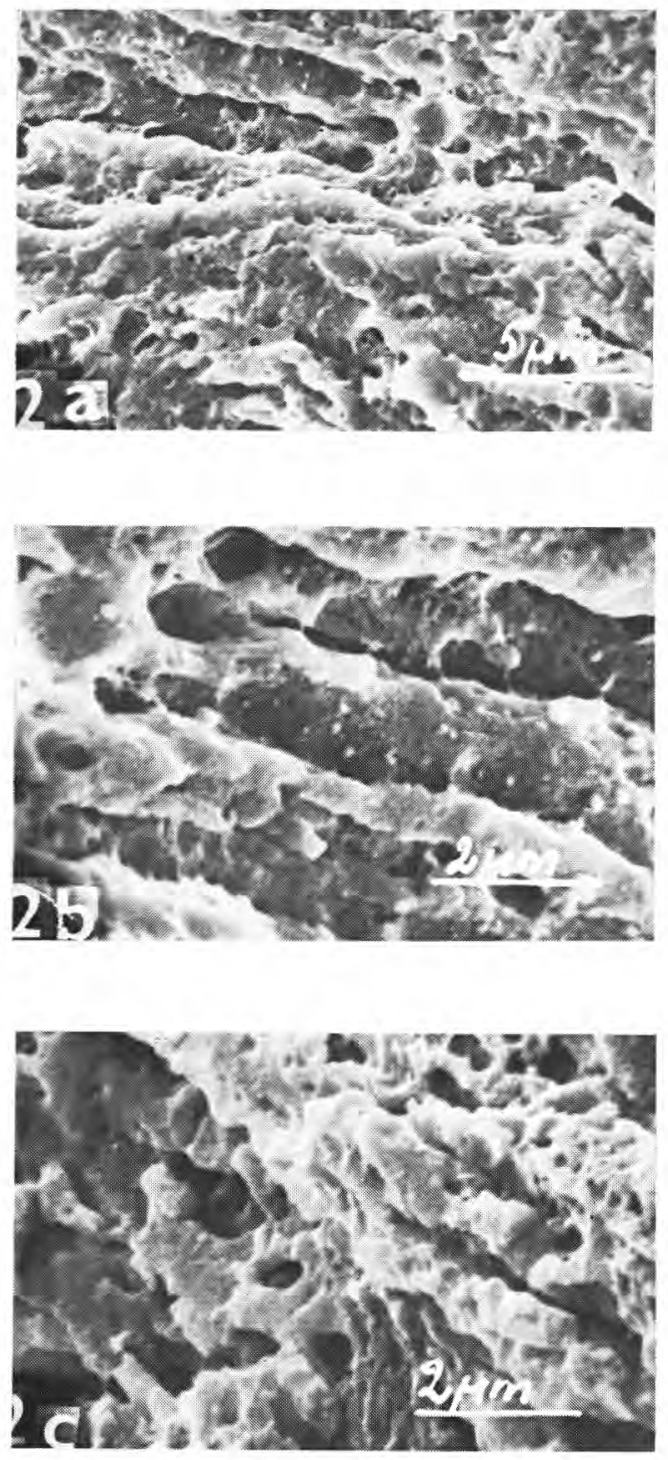

fig. 1

(2a, 2b, 2c)

Structure de la protéine texturée de lait.

que le texturat de soja contenait 2,30/16 g $\mathrm{N}$ d'acides aminés sulfuriques et $6,30 / 16 \mathrm{~g} \mathrm{~N}$ de lysine. La valeur biologique de la protéine du texturat de lait est plus élevée que celle des protéines texturées de soja. L'indice PER est de 2,1-2,2 pour des extrudats de soja et 1,8 pour des texturats obtenus par la méthode de filature [3]. 


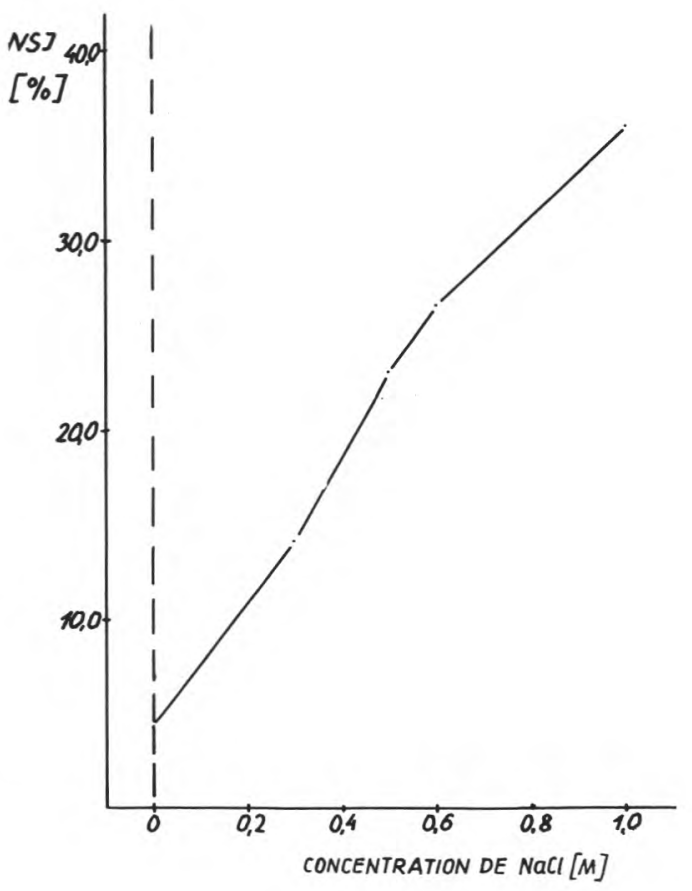

fig. 2

Influence de la concentration de $\mathrm{NaCl}$ sur l'indice de solubilité des matières azotées (NSI) du texturat.

\section{MICRO-STRUCTURE DU TEXTURAT}

Le tableau de micro-structure de la préparation, obtenu à l'aide du scanning microscope, nous présente des fibres qui se rangent parallèlement les unes à côté des autres (fig. 1-2 a, 2 b, 2 c). Dans quelques secteurs, plusieurs fibres plus minces sont réunies en une fibre plus épaisse. Des fibres individuelles ont une épaisseur variable sur toute leur longueur et l'on observe des renflements et des rétrécissements. Les fibres ont une forme cylindrique. Entre les fibres, des particules séparées de protéines sont rencontrées qui ne sont pas incluses dans des fibres.

Les observations microscopiques révèlent que la structure des protéines texturées de lait présente un caractère fibreux.

\section{PROPRIETES PHYSICO-CHIMIQUES}

L'indice NSI pour la protéine de texturat est de 4,3 p 100 (fig. 2) et il augmente sous l'action de la concentration croissante en $\mathrm{NaCl}$. 


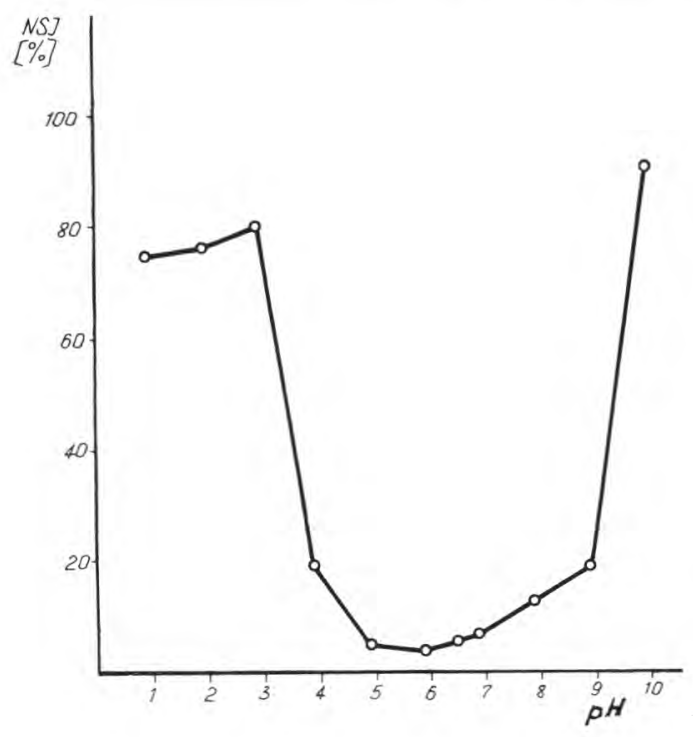

fig. 3

Influence du $\mathrm{pH}$ sur l'indice de solubilité des matières azotées (NSI) du texturat.

La relation entre l'indice NSI et la concentration de sel est à peu près directement proportionnelle dans l'intervalle de concentration de $\mathrm{NaCl}$ étudié. Il semble que la solubilité accrue des protéines, influencée par l'action de $\mathrm{NaCl}$, peut être expliquée par l'association des ions $\mathrm{Cl}^{-}$aux groupes actifs des chaînes protéiques. La charge totale des particules de protéine devient plus négative et les forces de refoulement qui agissent font accroître la solubilité de la protéine.

On a constaté que la protéine de texturat se caractérisait par la solubilité la plus basse dans l'intervalle de $\mathrm{pH}$ 5,0-6,0 (fig. 3). Nombre d'auteurs $[5,6]$ soulignent l'importance de la solubilité élevée des préparations protéiques, en considérant cette propriété comme celle qui détermine l'intérêt des préparations en tant que succédanés de la viande. Cependant, des préparations protéiques du type d'isolats et de texturats, qui se distinguent par une faible solubilité de leurs protéines, peuvent être utilisées dans l'industrie de la viande comme additifs, succédanés [8]. Malgré la faible solubilité de leur protéine, les préparations de ce type se caractérisent pourtant par de bonnes propriétés qui participent dans la formation de la structure, et elles lient bien la matière grasse et l'eau [16]. En cas des protéines texturées de lait, il semble que la valeur relativement basse de l'indice NSI détermine, d'une part, le type de la préparation, tandis que, d'autre part, elle fournit la preuve que cet indice ne peut être considéré comme le seul critère de l'intérêt d'une préparation en tant qu'additif aux produits de charcuterie et aux plats cuisinés. 


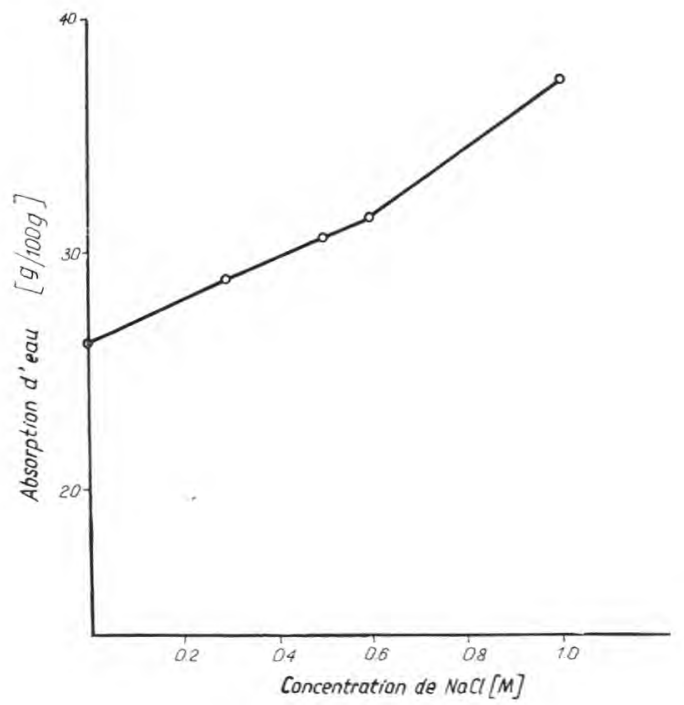

fig. 4

Influence de la concentration de $\mathrm{NaCl}$ sur l'absorption d'eau du texturat.

Cette thèse peut être confïrmée par le fait que des protéines texturées de lait présentent une bonne absorption d'eau $-26 \mathrm{~g}$ d'eau/ $100 \mathrm{~g}$ de préparation (fig. 4) qui augmente jusqu'à $37,7 \mathrm{~g}$ pour $100 \mathrm{~g}$ de préparation à la concentration de $1,0 \mathrm{M}$ en $\mathrm{NaCl}$.

La modification dans l'absorption d'eau sous l'influence du $\mathrm{NaCl}$ ajouté peut suggérer que l'interaction électrostatique entre ces chaînes protéiques est bien responsable du phénomène d'hydratation des protéines de la préparation.

Le pouvoir émulsif du texturat, exprimé en $\mathrm{V}_{100}$, décroît à mesure que la teneur en protéine de la solution augmente, cette valeur étant maintenue à un niveau constant pour une concentration de protéines située dans l'intervalle de $200-220 \mathrm{mg} / 10 \mathrm{ml}$ jusqu'à $300 \mathrm{mg} / \mathrm{ml}$ (fig. 5). L'allure de la courbe de relation entre le pouvoir émulsif, exprimé en pourcentage de la phase oléique (FO p. 100) et la teneur en protéines de la solution est inverse. D'abord, la valeur de FO p. 100 augmente, pour aboutir au maximum qui correspond à la teneur en protéines de $20 \mathrm{mg} / 10 \mathrm{ml}$ de la solution. L'étude sur l'aptitude à émulsifier la matière grasse, pour des valeurs diverses de $\mathrm{pH}$, a mis en évidence (fig. 6) que le texturat en solution de $\mathrm{NaCl}$ à $0,5 \mathrm{M}$ a le pouvoir émulsif le plus élevé à $\mathrm{pH} 6$, tandis que dans le milieu aqueux, pour la même acidité active, la préparation se caractérise par un pouvoir émulsif plus bas.

On a observé la similitude entre l'allure de la courbe qui représentait la relation du pouvoir émulsif du texturat, mesuré dans le milieu aqueux à un $\mathrm{pH}$ déterminé du milieu, et celle qui représentait la relation de la solubilité de la protéine dans la solution aqueuse à ce 


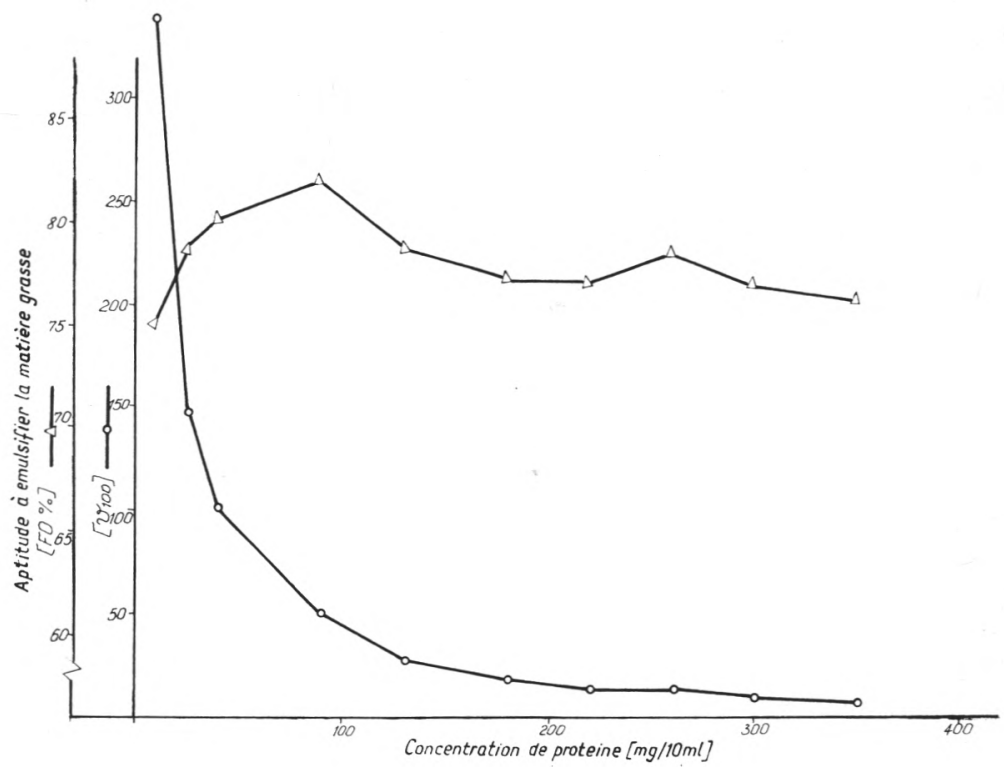

fig. 5

Influence de la concentration de protéine sur l'aptitude du texturat à émulsifier la matière grasse, exprimée en $\mathrm{V}_{100}$ et PO p. 100 .

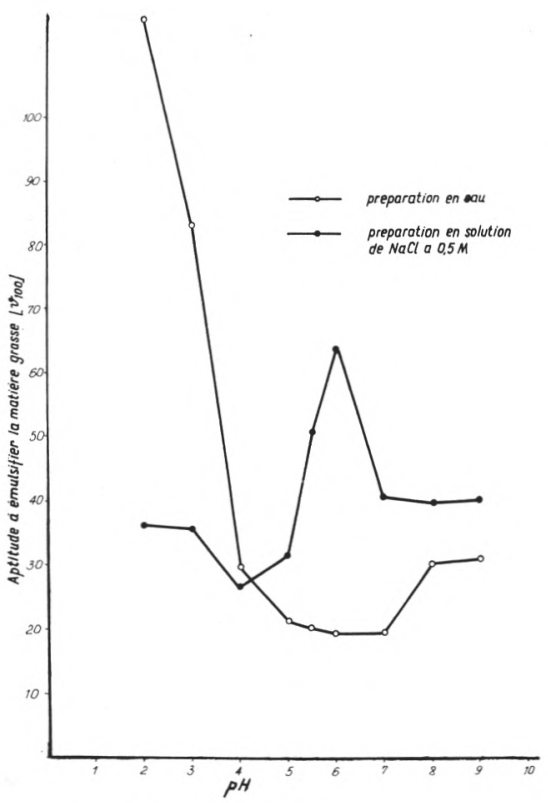

fig. 6

Influence du pH sur l'aptitude du texturat à émulsifier la matière grasse. 


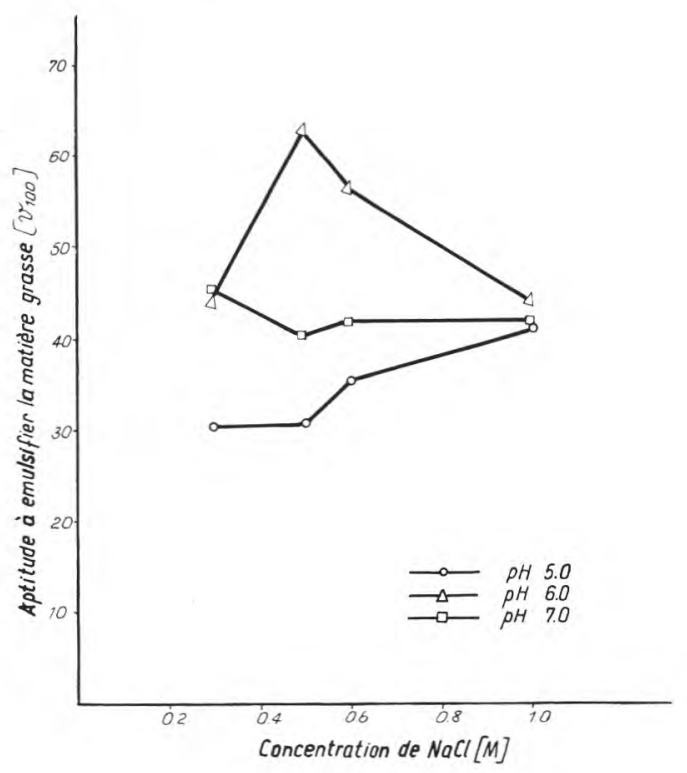

fig. 7

Influence de la concentration de $\mathrm{NaCl}$ sur l'aptitude du texturat à émulsifier la matière grasse.

$\mathrm{pH}$ du milieu. Des relations semblables ont été constatées par Kwasniewska et al. [8] pour la Promine R. Ces relations constatées laissent suggérer que la quantité de protéine, dissoute dans la solution, détermine le pouvoir émulsif du texturat dans le milieu aqueux, pour l'intervalle du $\mathrm{pH}$ étudié.

Le pouvoir émulsifiant le plus élevé du texturat se manifestant dans la solution de $\mathrm{NaCl}$ à $0,5 \mathrm{M}$, à $\mathrm{pH} 6$, ce fait semble indiquer que les protéines qui restent dissoutes dans les conditions décrites ont un pouvoir émulsifiant plus élevé que les protéines qui ont été dissoutes à des valeurs de $\mathrm{pH}$ supérieures ou inférieures à $\mathrm{pH} 6$.

La relation du pouvoir émulsifiant de la préparation pour la concentration de $\mathrm{NaCl}$ dépend de l'acidité active de milieu. Le pouvoir émulsifiant le plus élevé était observé à $\mathrm{pH}$ 6,0 et à la concentration $0,5 \mathrm{M}$ de $\mathrm{NaCl}$ (fig. 7).

\section{CONCLUSIONS}

Les résultats obtenus permettent de formuler les conclusions suivantes :

1. Les protéines texturées de lait, qui ont été produites par le procédé thermoplastique de fibrisation, se caractérisent par une valeur nutritive élevée qui correspond à la valeur nutritive de la caséine. 
2. Les protéines texturées de lait se révèlent être de structure fibreuse.

3. La solubilité des protéines du texturat dépend de la concentration en $\mathrm{NaCl}$ et du $\mathrm{pH}$ du milieu. La protéine de texturat se caractérise par la solubilité la plus basse à pH 5,0 et 6,0. L'absorption d'eau la plus élevée des protéines de lait a été constatée dans la solution de $\mathrm{NaCl}$. Le pouvoir émulsifiant de la préparation dépend de la teneur de la solution en protéines, du $\mathrm{pH}$ de milieu et de la concentration en $\mathrm{NaCl}$.

\section{Rés u m é}

La valeur nutritive de la protéine a été déterminée dans des protéines texturées de lait, qui étaient fabriquées par le procédé thermoplastique de fibrisation. Outre cela, la microstructure de la préparation a été également étudiée. Les propriétés physicochimiques choisies du texturat ont été caractérisées, l'influence de facteurs du milieu tels que, la concentration de la préparation, la valeur du $\mathrm{pH}$ et la concentration en $\mathrm{NaCl}$, étant surtout pris en considération. On a constaté que la protéine de la préparation se distingue par une valeur nutritive élevée et une structure de nature fibreuse.

\section{S u m m a r y}

\section{CHARACTERISTICS OF TEXTURED MILK PROTEINS}

A technique for production of textured milk protein, by thermoplastic fibrization procedure, was developed and spraid in industry.

The textured milk proteins are characterized by their high nutritive value (NPU $=61,2, \mathrm{PER}=2,6$ ) and by their high content of lysin, while their content of sulphuric amino acide remoins unchanged as compared to the raw material.

The scanning microscope studies indicate that the structure of textured milk proteins shows the fibrous nature.

Good structural properties of the texturate ensure a high fat emulsifyig ability $(200-300 \mathrm{~g} / 10 \mathrm{ml})$ and a good water binding ability (26/100 $\mathrm{g}$ of texturate) which increased to $37,8 \mathrm{~g} / 100 \mathrm{~g}$ of texturate at the $\mathrm{NaCl}$ concentration of $1.0 \mathrm{M}$.

The properties of textured milk proteins indicate that the new preparation may be considered to be the best meat substitute among all plant and animal protein texturates as known up to this day. The textured milk proteins are distinguished by the high heat stability of their fibrous structure. 


\section{Bibliographie}

[1] Budslawski (J.) (1967). - Metody analizy zywnosci. WPLiS Warszawa.

[2] Chapman (D. G.), Castillo (R.), Campbell (J. A.) (1959). - Evaluation of proteins in foods. P.I. Canad. J. Bioch. Physiol., 37, 679.

[3] Hamdy (M. M.) (1974). - Nutritional aspekts in textured soy proteins. J. Am. Oil Chem. Soc., 51, 85.

[4] Hayat (M. A.) (1977). - Principles and techniques of electron microscopy. Van Nostrand Reinhold Company.

[5] Hermansson (A. M.) (1973). - Determination of functional properties of protein foods. In: Proteins in human nutrition, chapter 27. Ed. Porter J.W. G., Rolls B. A., Academic Press, London.

[6] Inclaar (P. A.), Fortuin (J.) (1969). - Determining of emulsifying and emulsion stabilizing capacity of protein meat additives. Food Technol., 23, 103.

[7] Janicki (M. A.), WalczaK (Z.) (1954). - Water content of meat and its determination methods. Przem. Roln. $i$ Spoz., 8/6, 17, in polish.

[8] KWASNiewska (I.), Jacorzynski (B.), Barylko-Pikielna (N.) (1976). - Emulsification capacity characteristics of some soyabean protein preparations. Acta Alimentaria Polonica, 2/26, 307.

[9] Miller (D. S.), Bender (A. E.) (1955). - The determination of the net utilization of proteins by a shortened method. Brit. J. Nutr., 9, 382.

[10] Mitchell (H. H.), Block (R.J.) (1946). - Some relationship between the amino acid content of protein and their nutritive values for the rat. J. Biol. Chem., 163, 599.

[11] Moore (S.), Spackman (D. H.), Stein (W. H.) (1958). - Chromatography of amino acids on sulfonated polystyrene resins. Anal. Chem., 30, 1185.

[12] Opienska-Blauth (J.), Charezinski (M.), Berbec (H.) (1963). - A new rapid method of determining tryptophan. Anal. Bioch. 6, 69.

[13] Oser (B. L.) (1951). - Method for integrating essential amino acid content in the nutritional evaluation of protein. J. Am. Dietetic Ass., 27, 396.

[14] Poznanski (S.), Smietana (Z.), Szpendowski (J.), Stypulkowski (H.), Janicki (J.), SzewCZYK (Z.). - Method of production of thermostable textured milk proteins. UP. USA 891, 493 (1978), UP. DDR 136, 330 (1978), Polish Patent P - 197, 104 (1978).

[15] Schram (E.), Moore (S.), Bigwood (E. J.) (1954). - Chromatographic determination of cystine as cysteic acid. Biochem. J., 57, 33.

[16] Smith (G. C.), Juhn (H.), Carpentier (Z. L.), Mattil (K. F.), Cater (M.) (1973). - Efficacy of protein additives as emulsion stabilizers in frankfurters. J. Food. Sci., 41, 1148.

[17] Spackman (D. H.), Stein (W. H.), Moore (S.) (1958). - Automatic recording apparatus for use in the chromatography of amino acids. Anal. Chem., 30, 1190.

[18] Spies (J. R.), Chambers (D. C.) (1949). - Chemical determination of tryptophan in proteins. Anal. Chem., 21, 1249.

[19] Swift (C. E.), Lockett (C.), Tryar (A. J.) (1961). - Comminuted meat emulsions - the capacity of meats for emulsifying fat. Food Technol., 15, 468. 